Revue des patrimoines

\title{
L'archipel des coupoles. Topographie et architecture de l'observatoire de Toulouse : les logiques historiques et patrimoniales du bâti scientifique
}

Jérôme Lamy et Béatrice Motard

\section{(2) OpenEdition}

Journals

Édition électronique

URL : http://journals.openedition.org/insitu/3729

DOI : $10.4000 /$ insitu.3729

ISSN : 1630-7305

Éditeur

Ministère de la culture

Référence électronique

Jérôme Lamy et Béatrice Motard, "L'archipel des coupoles. Topographie et architecture de

l'observatoire de Toulouse : les logiques historiques et patrimoniales du bâti scientifique », In Situ [En

ligne], 10 | 2009, mis en ligne le 19 mai 2009, consulté le 01 mai 2019. URL : http://

journals.openedition.org/insitu/3729 ; DOI : 10.4000/insitu.3729

Ce document a été généré automatiquement le 1 mai 2019.

\section{(i) $\odot$

In Situ Revues des patrimoines est mis à disposition selon les termes de la licence Creative Commons Attribution - Pas d'Utilisation Commerciale - Pas de Modification 4.0 International. 


\title{
L'archipel des coupoles. Topographie et architecture de l'observatoire de Toulouse : les logiques historiques et patrimoniales du bâti scientifique
}

\author{
Jérôme Lamy et Béatrice Motard
}

1 Le bâti scientifique constitue un objet patrimonial original et peu étudié. Nous nous proposons d'abord ici de mettre au jour les origines de l'agencement topographique et architectural de l'observatoire de Toulouse. Peter Galison a montré l'intérêt qu'il y avait à analyser la manière dont la structure des bâtiments savants pouvait configurer l'activité scientifique et inversement, comment la pratique savante redessinait les formes, les surfaces et les volumes des monuments ${ }^{1}$.

2 Nous examinerons donc, dans un premier temps, la genèse et l'évolution architecturale de l'observatoire toulousain, tout au long du XIXe siècle. Notre étude prendra ensuite appui sur cette histoire singulière du lieu pour explorer les voies d'une possible valorisation patrimoniale. Nous détaillerons notamment les relations dialectiques que doivent entretenir la mise en valeur du site et la recherche historique.

\section{1 - La fondation d'un nouvel observatoire toulousain au XIX' siècle}

3 Toulouse est, au XVIII ${ }^{e}$ siècle, un centre scientifique de première importance. Jérôme Lalande, hiérarque de l'astronomie française, assure que la cité garonnaise est « de toutes les villes des province, celle où l'astronomie a été le plus cultivée $»^{2}$. Les deux astronomes majeurs de Toulouse au siècle des Lumières, Antoine Darquier et François Garipuy, ont fondé leurs propres observatoires au sommet de leurs maisons. Lorsque Garipuy meurt en 
1782, l'Académie des sciences toulousaines protège et occupe cet espace savant jusqu'à la Révolution ${ }^{3}$. L'observatoire échappe au vandalisme et à la destruction grâce notamment à l'intervention du Bureau des longitudes qui, en 1796, interdit que le bâtiment soit compris parmi les biens nationaux à vendre ${ }^{4}$. Dans le premier tiers du XIX ${ }^{\mathrm{e}}$ siècle, les astronomes qui se succèdent à la tête de l'institution astronomique désormais prise en main par la municipalité contribuent à l'élaboration d'un discours mythifiant l'observatoire. L'espace savant est valorisé pour son âge d'or passé et il fait l'objet d'un respect scrupuleux qui interdit tout changement dans l'architecture ou dans l'agencement des lieux ${ }^{5}$ (fig. $\left.\mathbf{n}^{\circ} \mathbf{1}\right)$.

Figure 1

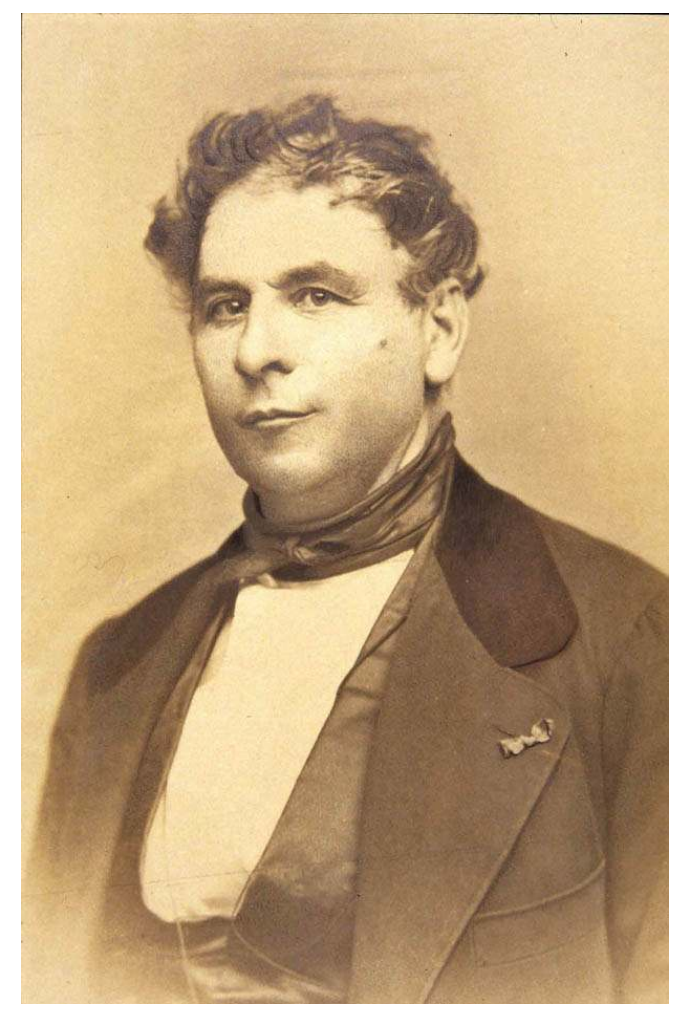

Portrait de Frédéric Petit (1810-1865). Tirage photographique fin XIXe siècle de Fac [Facchineti], d'après un tirage original de E. Delon réalisé vers 1860. Papier bromure 35,5 x 48,5 monté sur carton $47 \times 63$. Archives municipales de Toulouse, cote 7 Z (fonds de l'observatoire).

Ces égards à l'endroit de l'observatoire de Garipuy cessent avec l'arrivée de Frédéric Petit. Cet astronome est un élève du célèbre savant parisien François Arago qui l'a imposé à Toulouse en 1838. Petit refuse de prendre à son compte le mythe jusqu'ici entretenu d'un établissement savant dont l'importance repose uniquement sur une histoire glorieuse. Lorsqu'il prend possession des lieux, il écrit à son collègue parisien Alexis Bouvard pour lui décrire l'observatoire. Petit assure que «tout y a été fait au Rebours du Bon sens ${ }^{6}$. La terrasse est peu commode et mal protégée. Enfin, les murs sont « dilaté[s] tout à fait irrégulièrement par le Soleil ou mis en vibration par le mouvement des voitures ${ }^{7}$.

Le nouvel astronome ne se contente pas de critiquer un bâtiment qu'il juge obsolète pour l'observation, il propose immédiatement au maire de Toulouse la translation $^{8}$ de l'établissement scientifique. Les édiles municipaux ne sont d'abord guère enthousiasmés par ce projet ambitieux et onéreux. Lors d'une séance du conseil municipal, l'un des 
membres manifeste avec virulence sa désapprobation et conteste «l'impossibilité prétendue de construire dans l'enceinte de l'observatoire $»^{9}$ de nouveaux aménagements. François Arago soutient son élève et propose d'aider la ville dans l'établissement d'une institution astronomique. "La ville savante du midi a grandement raison de vouloir posséder un véritable observatoire $»^{10}$, assure l'astronome parisien. Ces encouragements d'Arago galvanisent les énergies toulousaines.

Le conseil municipal met donc au concours le projet de construction d'un observatoire en décembre $1839^{11}$. Frédéric Petit n'apprécie guère cette méthode et met en garde les édiles; il assure qu'Arago n'a pas « soupçonné un seul instant que ce concours quoique bon dans son principe, put être appliqué à ce monument $»^{12}$. Petit ne souhaite pas que les plans du nouvel observatoire se fassent sans son avis ni celui d'Arago. Les deux astronomes vont d'ailleurs tenter peu à peu de prendre l'ascendant sur les édiles. Ils tiennent notamment à s'impliquer dans le choix de l'emplacement et la construction du bâtiment. Petit demande au maire, Armand Perpessac, en décembre 1839, de nommer " une commission chargée de rechercher avec [lui] l'emplacement le plus convenable » ${ }^{13}$. François Arago avait déjà précisé les conditions d'implantation d'un observatoire astronomique. Le savant préconisait «l'éloignement [qui], loin d'être un inconvénient serait, à [s]es yeux un avantage : l'observatoire n'aurait plus alors à redouter les visites quotidiennes de ces nuées de désœuvrés qui abondent dans les villes populeuses et deviennent la désolation de quiconque sait faire un bon usage de son temps. Un observatoire ne doit pas être assimilé à un musée, à une galerie de tableaux : l'astronome entouré du public, ne peut se livrer à aucune observation utile $»^{14}$. L'observatoire n'est pas un bâtiment comme les autres. Les astronomes, tels des anachorètes, souhaitent travailler à l'écart de la vie urbaine, dans leur thébaïde savante. L'orientation géographique du lieu est également importante, et Arago précise au premier magistrat de la cité garonnaise qu'il ne faut pas établir le monument «sur le penchant d'une colline. Un point culminant vaudrait beaucoup mieux, dût-on aller le chercher plus loin de Toulouse $»^{15}$. C'est finalement le coteau des redoutes ${ }^{16}$, à l'est de la cité garonnaise, qui est désigné.

7 Les discussions s'organisent désormais autour de l'architecte de la ville, Urbain Vitry. Formé à l'École des arts de Toulouse, il a également suivi les cours du baron Charles Dupin au Conservatoire des arts et métiers ${ }^{17}$. Les conseillers municipaux le chargent de "s'entendre avec M. Petit à l'effet de rédiger le projet d'un nouvel observatoire " ${ }^{18}$. Frédéric Petit tient à dissiper d'éventuels malentendus et précise les termes mêmes de la collaboration des astronomes avec Urbain Vitry. Il précise donc aux édiles la place de chacun dans le projet et met en garde contre «l'idée commune que la construction de cet édifice ne présenterait pas plus de difficultés que celle de tout autre monument $»^{19}$. Petit assure que la "spécialité d'un tel établissement ne permet de donner un programme convenable, sans juger de la grandeur, de la position, et en quelque sorte de la composition la plus intime de toutes les parties de l'édifice, tous les plans devraient donc se ressembler et être une expression fidèle des idées de l'astronome qui les aurait inspirées $»^{20}$. La première ébauche du bâtiment a été imaginée «dans [s]es nombreuses conférences avec $\mathrm{M}$. Vitry ${ }^{21}$. Frédéric Petit développe son argumentaire en insistant sur les multiples échecs architecturaux, fruits d'un mépris des impératifs savants dans l'élaboration des observatoires de Paris, du Collège de France ou de Bruxelles. L'astronome toulousain rappelle au maire de la ville que «toutes les fois qu'on a voulu livrer à un architecte le plan d'un observatoire, l'on n'a eu que de vicieuses 
constructions ${ }^{22}$. La pratique savante définit un cahier des charges précis. Le plan s'ébauche en suivant les contraintes imposées par la science ; le bâtiment répond d'abord aux exigences de l'observateur. Dès lors, l'architecte est relégué au second plan. Petit reconnait qu'il « n'est en quelque sorte que le traducteur $»^{23}$ des idées de l'observateur. François Arago participe lui aussi à l'élaboration du projet. Il souhaite notamment que « tous les appartements fussent disposés au rez-de-chaussée ${ }^{24}$. Finalement, la réalisation du plan de l'observatoire est d'abord l'aboutissement des réflexions de Frédéric Petit et de son maître parisien qui parviennent à réduire le rôle d'Urbain Vitry à celui d'un technicien du dessin (fig. $\mathbf{n}^{\circ}$ 2).

Figure 2

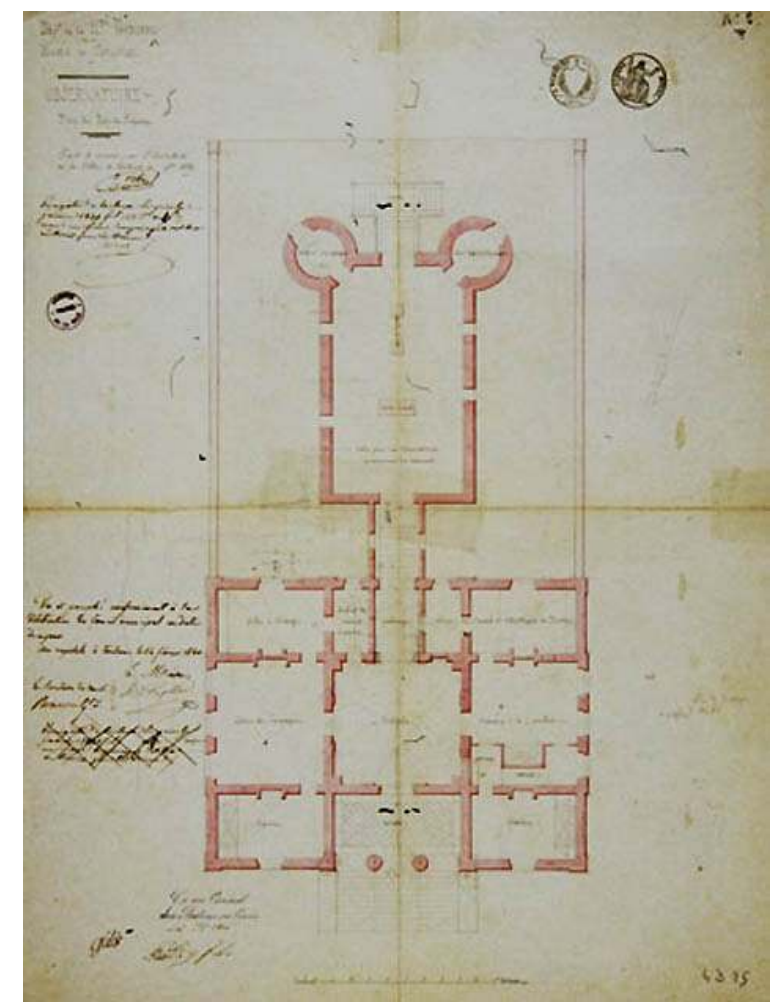

Observatoire de Toulouse. Plan du rez-de-chaussée daté novembre 1839 dressé et signé par Urbain Vitry, architecte de la ville, échelle : 1/100; plan aquarellé sur papier, $43 \times 55$. Archives municipales de Toulouse, cote 4 D 95.

Un premier devis descriptif est dressé en 1840, la dépense estimée et prise en charge par la Ville s'élève à 68000 francs $^{25}$ et les travaux sont confiés à l'entrepreneur Gilis ${ }^{26}$. La prégnance des astronomes dans les phases préparatoires se prolonge pendant la construction du bâtiment. Petit écrit au maire de Toulouse en mai 1843 pour lui indiquer qu'il serait indispensable de prévoir des massifs en pierre de taille pour les outils scientifiques, afin de réduire les oscillations ${ }^{27}$. Michèle Eclache a comparé les plans initiaux et la réalisation architecturale finale. Elle souligne en particulier «une augmentation générale des volumes au-dessus du sol, surtout sensible pour le bâtiment des observations où elle se réalisa par un aplanissement du terrain qui permit la création d'un rez-de-chaussée sous la salle des instruments ${ }^{28}$. 》 (fig. $\mathbf{n}^{\circ} \mathbf{3}$ ) 


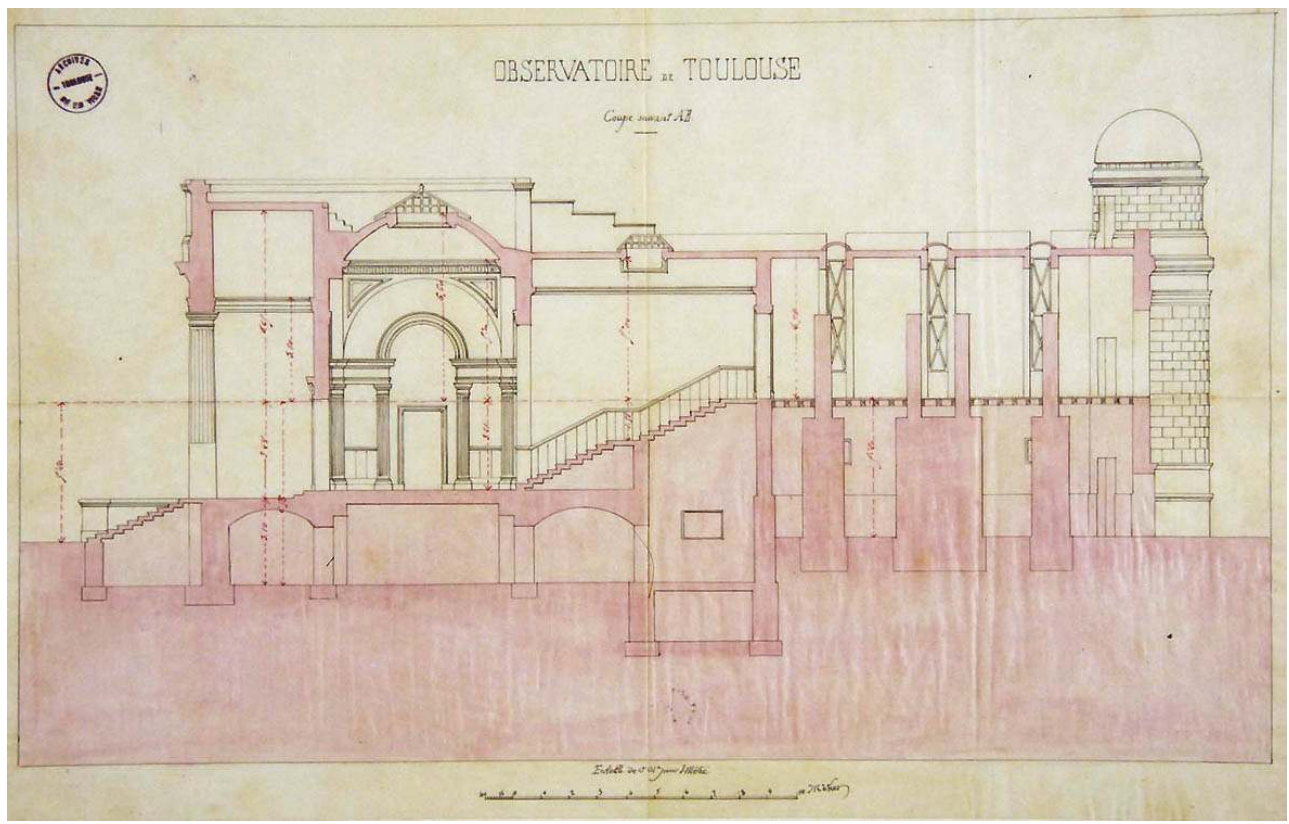

Observatoire de Toulouse. Coupe aquarellée sur calque vers 1860 ; échelle : 1/100. 47 × 30. Archives municipales de Toulouse, non coté.

9 La construction de l'édifice astronomique connaît de nombreux retards. Urbain Vitry, victime d'une disgrâce à la suite d'un renversement de majorité du conseil municipal en 1843, est contraint de quitter ses fonctions. L'entrepreneur Gilis est directement mis en cause pour la lenteur des travaux. Le maître d'œuvre se défend en expliquant que le retard est imputable aux « changements et augmentations qu'a subis le plan primitif ${ }^{29}$, aux hésitations des édiles, ainsi qu'à tous les imprévus auxquels il a dû faire face. L'observatoire est achevé en 1844. La dépense finale pour l'établissement de l'espace savant est de 120518 francs, soit plus du double du budget prévu ${ }^{30}$.

Le bâtiment est scindé en deux parties distinctes, mais reliées par un passage. La première rassemble les appartements du directeur et l'ensemble des pièces d'habitation, alors que la seconde est dévolue à la pratique scientifique. La rupture entre les deux blocs architecturaux contigus est rendue "visible de l'extérieur par l'utilisation d'un style différent $»^{31}$. Le logement s'étend sur deux étages et comprend des chambres, un cabinet de travail, une bibliothèque, une salle à manger, un salon de compagnie, ainsi qu'un vestibule monumental. Marie-Laure Capella indique que « la décoration intérieure frappe par une grande sobriété traduisant toute la rigueur et un certain académisme qui se lient aux lieux d'études ${ }^{32}$ ». L'organisation de la partie destinée à l'observation est plus réduite et tient compte des contraintes instrumentales. Les soubassements aménagés sont en effet particulièrement importants. Frédéric Petit a prévu «deux forts piliers en maçonnerie pour la lunette méridienne et un pilier pour le cercle mural $\aleph^{33}$. L'ensemble architectural est complété par deux tourelles dont l'une comporte un toit tournant. Le porche d'entrée est conçu sur un mode monumental avec les deux colonnes cannelées à chapiteau dorique, « soutenant un large entablement $»^{34}$. Comme l'a souligné Marie-Laure Capella, l'observatoire est conforme à l'inspiration néoclassique d'Urbain Vitry et évoque ses autres réalisations architecturales toulousaines, comme l'École de médecine ${ }^{35}$. 
11 Frédéric Petit ne s'est pas seulement impliqué dans l'organisation architecturale de l'observatoire, il a également veillé à protéger l'espace savant de l'expansion urbaine. L'astronome s'emploie à soustraire son bâtiment à la curiosité des foules et à l'extension de la ville. Il écrit au maire de Toulouse en 1848 pour lui suggérer de clôturer l'ensemble des bâtiments. Il justifie l'importance d'une telle construction en insistant sur «[le] recueillement et [le] silence que réclament les observations astronomiques, très souvent troublées par des enfants ou la curiosité des promeneurs $»^{36}$. Le conseil municipal accède à cette demande et constate «qu'il y a lieu de clore les terrains dépendants de cet établissement par un mur de ceinture $»^{37}$ (fig. $\left.\mathbf{n}^{\circ} \mathbf{4}\right)$.

Figure 4

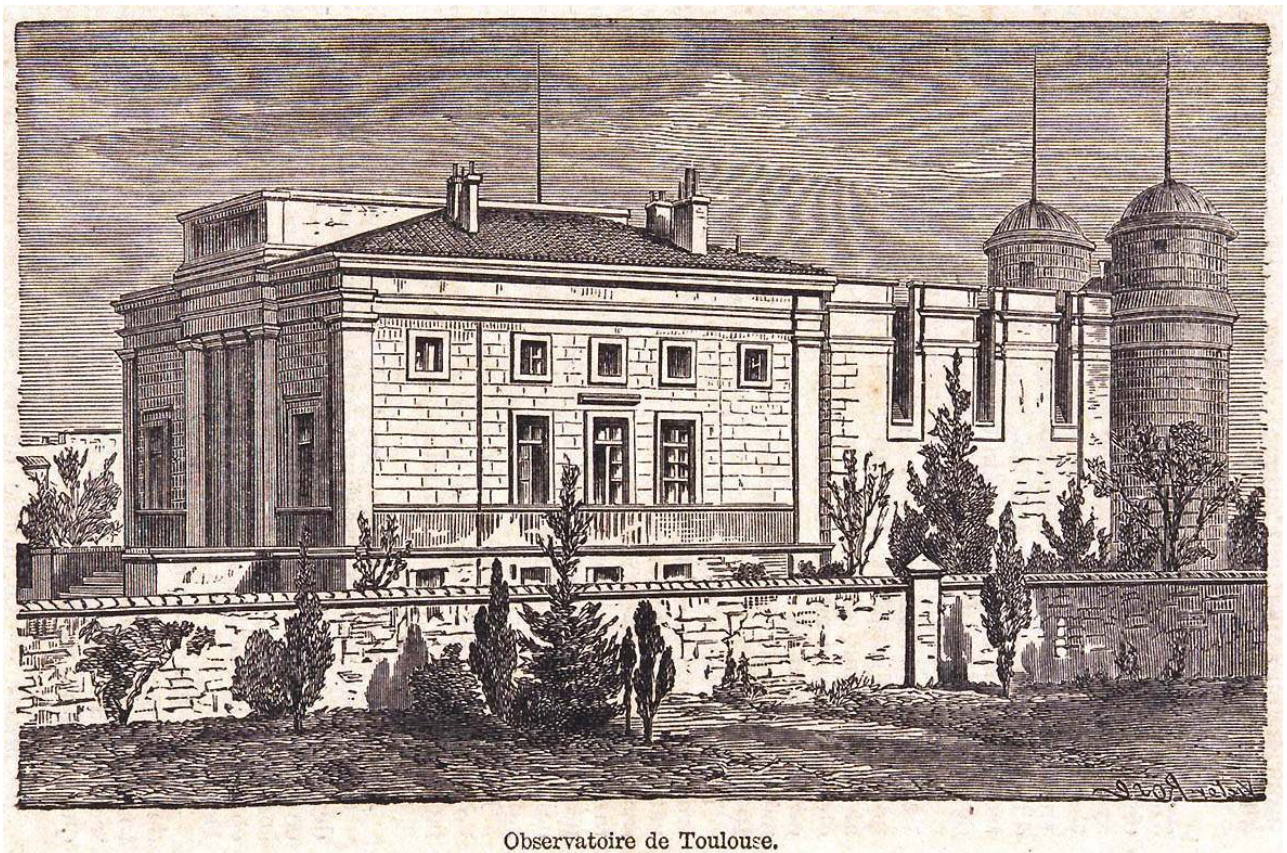

Gravure de l'observatoire de Toulouse dans Guide illustré dans Toulouse et dans le département de la Haute-Garonne, Toulouse, Imprimerie Paul Privat, 1877, in-16; p. 143-144. Gravure d'après un dessin de Victor Rose. Bibliothèque des Archives municipales de Toulouse.

Il ne s'agit pas seulement d'un repli de l'observatoire sur lui-même, mais également d'une neutralisation de l'environnement immédiat de l'édifice, transformé en zone non aedificandi. Dès 1843, Petit s'inquiète de voir s'établir un pigeonnier aux abords de l'espace savant. L'astronome redoute les difficultés d'observation qu'entraînerait le surgissement d'une multitude de monuments à proximité de l'établissement scientifique. L'instrument méridien, en particulier, exige un horizon parfaitement dégagé. Petit suggère donc à la ville de devenir "propriétaire de toute la crête du mamelon sur lequel est bâti l'observatoire $»^{38}$. L'astronome n'ignore pas le mouvement d'expansion urbaine qui caractérise Toulouse au milieu du XIX ${ }^{\mathrm{e}}$ siècle. Il martèle qu'en « présence de l'impulsion qui entraîne la ville vers le coteau des Redoutes, il est hors de doute qu'avant peu des constructions et des plantations de toute espèce entoureront complètement l'observatoire ${ }^{39}$. Le successeur de Frédéric Petit à la tête de l'établissement scientifique, Pierre-Adolphe Daguin, adopte la même ligne de défense et mobilise les édiles en 1868. Ceux-ci reconnaissent que l'installation de cafés et de guinguettes près de l'observatoire serait « un voisinage gênant pour le Directeur $»^{40}$. La municipalité, bien que consciente 
des dangers présentés par les astronomes, réagit avec lenteur. En 1847 les édiles ont obtenu du roi Louis-Philippe un décret qui déclare « d'utilité publique le dégagement des lignes méridiennes de l'observatoire de la ville de Toulouse ${ }^{41}$. Cette première protection ne suffit pas et en 1868 , le maire Jean Filhol doit acheter des parcelles voisinant le lieu consacré aux sciences.

On mesure donc combien la construction de l'observatoire toulousain remodèle l'environnement et discipline le territoire avoisinant. Les zones proches de l'établissement astronomique sont neutralisées et le bâtiment lui-même est protégé par une ceinture repoussant d'éventuels curieux. La construction de l'observatoire se poursuit après l'édification du bâtiment principal.

\section{2 - L'extension du lieu de sciences}

14 L'astronomie française connaît un renouvellement institutionnel profond avec l'avènement de la III République. Des observatoires sont créés à Lyon, Bordeaux et Besançon. Ceux de Toulouse et de Marseille sont relancés. Une série de décrets organise le développement des carrières des astronomes, instaure une structure hiérarchisée et met en place des instances de gestion et de contrôle de l'activité scientifique. Le pouvoir républicain remodèle la cartographie de la science astronomique en France en même temps qu'il dote les différents établissements de moyens financiers conséquents ${ }^{42}$.

Depuis le milieu du XIX ${ }^{e}$ siècle, l'instrumentation scientifique connaît un changement important dans la dimension des outils. Dieter B. Hermann remarque que « la production industrielle d'instruments astronomiques s'est accrue au cours du XIX ${ }^{e}$ siècle, en relation étroite avec le développement des instruments de mesure, des outils et de l'équipement technique des autres sciences ${ }^{43}$. Les fabricants d'outils scientifiques s'attachent d'abord à développer les techniques relatives aux lunettes astronomiques. Albert Van Helden souligne que «la seconde moitié du XIXe siècle vit d'une part la dimension des lunettes croître jusqu'à la taille que nous leur connaissons aujourd'hui, et d'autre part apparaître le télescope moderne avec son miroir de verre à argenture métallique $»^{44}$. Les autres types d'instruments astronomiques profitent de ces transformations. L'astronomie française suit une évolution technique qui accroît la puissance des outils d'observation (fig. $\mathbf{n}^{\circ}$ 5). 
Figure 5

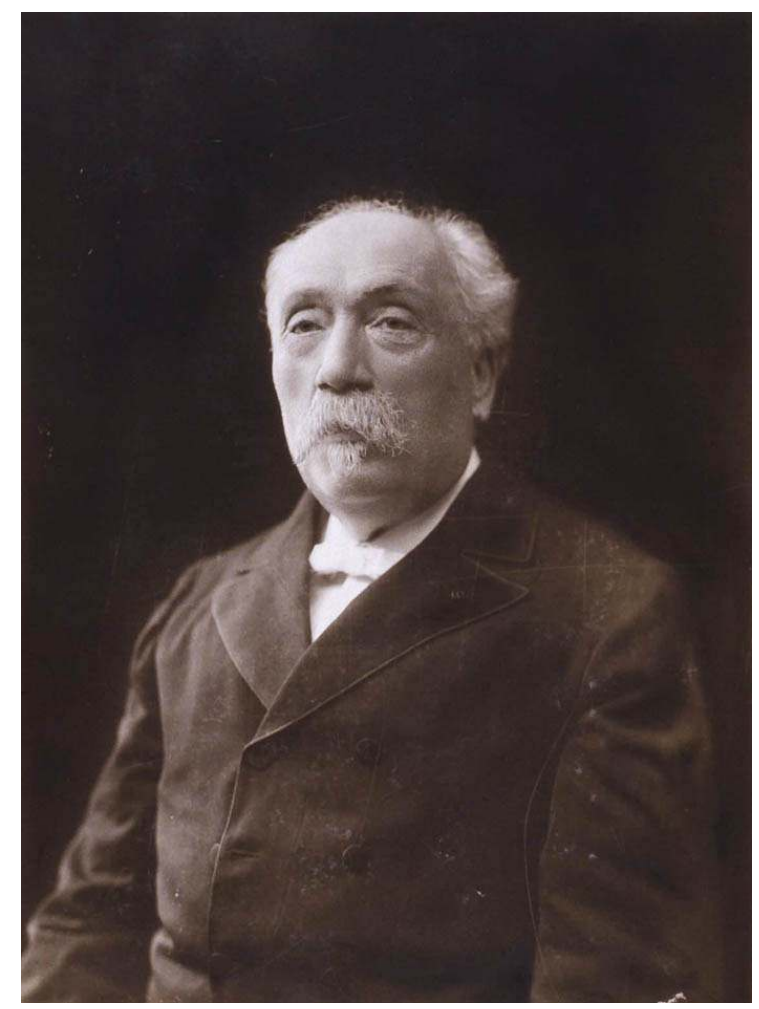

Portrait de Benjamin Baillaud (1848-1934). Cliché Eugène Pirou (Paris). Photographie 21 x 28 collée sur papier $36 \times 47$. Archives municipales de Toulouse, cote 7 Z (fonds de l'observatoire).

16 Félix Tisserand et Benjamin Baillaud, les deux directeurs nommés successivement après la réorganisation de l'établissement savant toulousain en 1872, s'emploient à compléter leur arsenal technique. Tisserand reçoit d'abord un télescope de $80 \mathrm{~cm}$, commandé par Petit et conçu sur le modèle de celui de l'observatoire de Marseille ${ }^{45}$. En 1876, il souhaite faire installer « un équatorial de neuf pouces d'ouverture $»^{46}$. Il demande un devis aux constructeurs parisiens Brunner. La dépense est évaluée à 60000 mille francs. Pour convaincre le ministre de l'Instruction publique de débloquer les fonds nécessaires à l'achat de l'instrument, Félix Tisserand souligne que «tout observatoire sérieux en possède un semblable ${ }^{47}$. Benjamin Baillaud, nommé directeur de l'observatoire de Toulouse en 1878, réclame un cercle méridien, afin "d'entreprendre des travaux de longue haleine, notamment la vérification et l'étude des catalogues d'étoiles multiples en vue de la détermination des mouvements propres de ces systèmes stellaires ${ }^{48}$. L'astronome insiste sur le fait qu'il existe une complémentarité entre l'équatorial et le cercle méridien. Ces deux outils forment un continuum technique et Baillaud soutient que l'achat du premier implique l'acquisition du second.

17 Le capital technique des observatoires français à la fin du XIX ${ }^{e}$ siècle semble homogène dans sa composition. L'idée selon laquelle chaque établissement doit posséder un corpustype d'instruments d'observation émerge peu à peu pendant cette période. Les équatoriaux et les cercles méridiens forment l'essentiel de l'équipement des observatoires. Celui de Toulouse y adjoint un télescope qui étoffe encore un peu plus le capital instrumental. En 1908, lors du départ de Benjamin Baillaud, l'établissement de la cité garonnaise renferme quatre instruments de grande dimension : un petit équatorial 
de Brunner installé en 1880, un télescope de $80 \mathrm{~cm}$, un équatorial photographique érigé en 1890 et un cercle méridien mis en place la même année ${ }^{49}$ (fig. $\mathbf{n}^{\circ}$ 6).

Figure 6

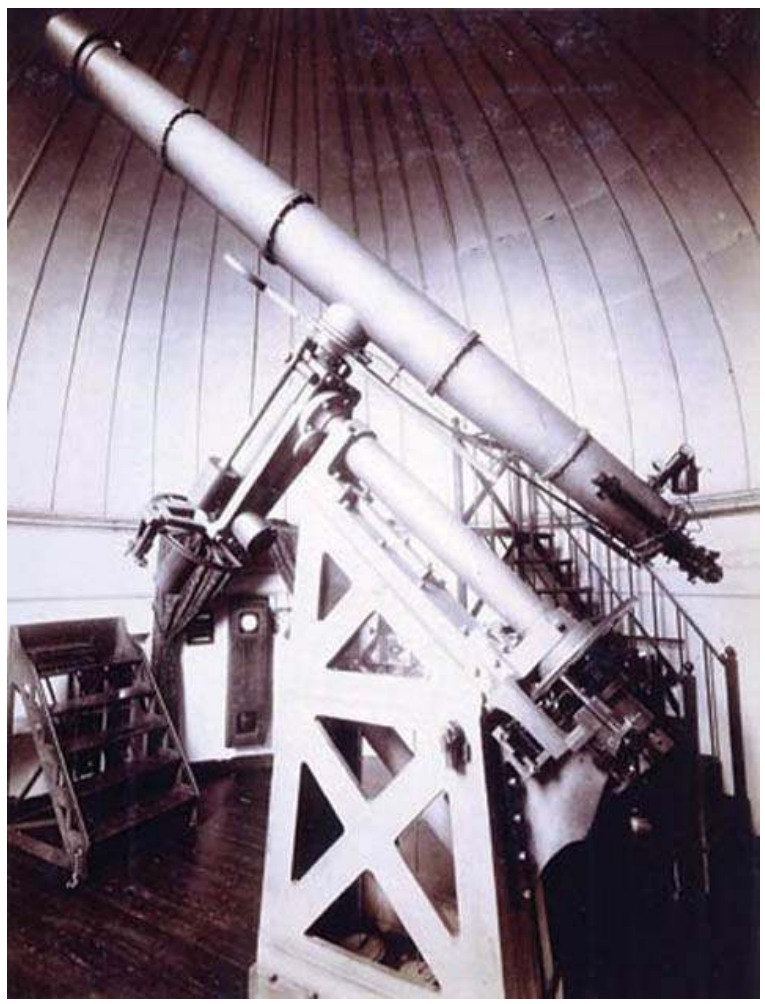

La lunette équatoriale de Brunner. In Observatoire de Toulouse, matériel ; Photographies des instruments et de leurs principaux détails par Mr Henry Bourget, docteur ès sciences et maître de conférence à l'Université et astronome-adjoint à l'Observatoire. Circa 1900. Photographie, 22 × 28 collée sur carton $44 \times 34$. Archives municipales de Toulouse, cote 7 Z (fonds de l'observatoire).

L'intrusion des instruments de grande dimension dans l'observatoire modifie l'agencement du lieu. Il est nécessaire, pour les placer convenablement, de trouver des espaces appropriés. Benjamin Baillaud relate en 1889 les efforts de son prédécesseur Tisserand pour obtenir de la Ville l'agrandissement des terrains au sud ${ }^{50}$. Cette extension de la surface de l'établissement permet de construire des coupoles ou des pavillons pour y abriter les outils scientifiques. L'observatoire est donc un espace fragmenté qui prend désormais la forme d'un archipel d'édifices consacrés à l'examen du ciel. Cette nouvelle distribution des ressources techniques accompagne une modification fondamentale de la pratique astronomique. Le régime d'organisation scientifique qui se met en place après 1870 repose sur une très forte division du travail, qui rapproche l'observatoire du modèle de l'usine, comme l'a montré Simon Schaeffer ${ }^{51}$. Chaque astronome est désormais exclusivement associé à un instrument, dont il dirige le maniement, secondé par un ou plusieurs assistants. Cette stricte séparation des tâches détermine, pour les observateurs, les objets célestes qu'ils vont étudier. Les caractéristiques de chaque outil scientifique fixent une gamme limitée d'observations possibles et circonscrivent le champ de recherche de celui qui le manipule. Le cercle méridien est par exemple destiné à «la formation d'un catalogue de trois mille six cents étoiles $\|^{52}$. La structuration de l'espace et l'organisation topographique du lieu participent d'un mouvement d'ensemble de la pratique scientifique désormais calquée sur le modèle industriel. 
L'architecture des coupoles répond à des impératifs précis. En 1885, le constructeur d'instruments Paul Gautier donne ses conseils à Benjamin Baillaud pour ériger un bâtiment adapté à l'astrographe : « Il n'y a aucun inconvénient à donner à la coupole [un] diamètre (...) [de] 6,50 $\mathrm{m}$. Vous pouvez également augmenter la hauteur de la Tour pourvu que son rapport avec le pilier soit le même ${ }^{53}$. L'aménagement du lieu doit permettre une protection optimale de l'instrument. La salle méridienne n'est pas une coupole, mais un bâtiment avec un toit s'ouvrant en deux parties au faîtage pour permettre le pointage de la lunette dans le plan méridien (plan nord-sud). Sa conception intègre les indispensables précautions qui concourent à l'optimisation maximum des conditions d'utilisation de l'outil. Baillaud explique en 1891 que cette pièce est " complètement achevée et qu'elle est revêtue sur toutes les faces d'un lambris en bois isolé des murs $»^{54}$ (fig. $\mathbf{n}^{\circ} \mathbf{7}$ ).

Figure 7

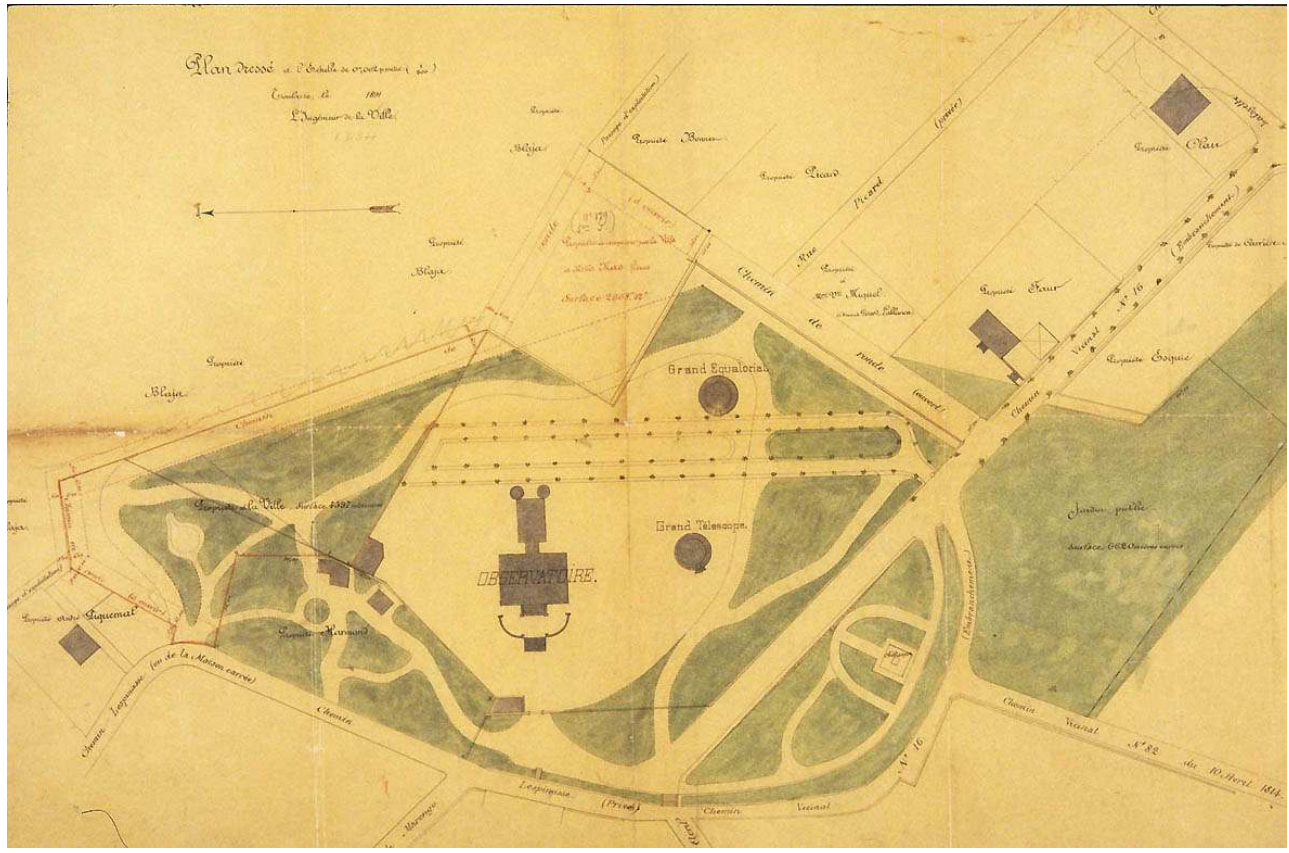

Plan de situation de l'Observatoire en 1891, plan aquarellé sur calque dressé par l'ingénieur de la ville ; 103,5 × 62 ; échelle: 1/500. Archives municipales de Toulouse, cote 4 D 577.

La construction des coupoles est confiée à des ouvriers toulousains et c'est un architecte de la Ville qui supervise les travaux. En mai 1876, Félix Tisserand signale au ministre de l'Instruction publique qu'en prévision de l'acquisition de l'équatorial, il a «fait faire un devis des dépenses pour la construction de la coupole de l'équatorial par M. Fitte, architecte de la Ville (...) depuis longtemps familiarisé avec les constructions de [l'] observatoire $\aleph^{55}$. L'érection des bâtiments destinés à recevoir les grands instruments témoigne d'une division des tâches particulière: les artistes parisiens fabriquent des objets sans contraintes architecturales, tandis que les maîtres d'œuvre de la cité garonnaise adaptent le bâti protecteur aux outils techniques grâce à une connaissance intime du projet scientifique qu'ils ont développé dans un dialogue permanent avec les astronomes. Comme lors de la construction du premier bâtiment au milieu du XIXe siècle, c'est l'ordre technique et instrumental qui préside à la conception des coupoles. 
Parallèlement à cet éclatement topographique de l'observatoire - éclatement nécessaire à la qualité des observations - le travail de domestication du paysage avoisinant le lieu de science, entrepris sous le Second Empire par Frédéric Petit, se poursuit avec Félix Tisserand et Benjamin Baillaud. Le dégagement des lignes méridiennes reste une préoccupation essentielle des directeurs de l'institution savante toulousaine. En 1891, le directeur de l'établissement souligne qu'il existe encore des parcelles vierges, à la merci de n'importe quel spéculateur susceptible d'ériger une maison avec une cheminée ${ }^{56}$. Dans le même temps, la recomposition spatiale imposée par l'introduction des instruments de grande taille et la construction des coupoles les enveloppant et le nécessaire espacement de ces différents éléments architecturaux pour la qualité des observations ne modifie pas la politique de claustration inaugurée par Frédéric Petit. L'agrandissement de l'espace d'observation repousse un peu plus loin le mur d'enceinte. Félix Tisserand exige en 1876 "le reculement du mur de clôture de l'observatoire, pour isoler complètement le grand télescope déjà établi et l'équatorial qui le sera probablement au mois de février [1877] $\aleph^{57}$. Le conseil municipal saisi de cette demande, reconnait "qu'il importe (...) d'isoler complètement les instruments (...) afin que $\mathrm{M}$. le directeur ne puisse pas être dérangé dans ses observations astronomiques $\aleph^{58}$. La croissance de l'établissement dans la dernière partie du XIX siècle contraint à une incessante redéfinition des frontières matérielles. Benjamin Baillaud propose à la ville d'étendre la surface de son établissement pour lui donner « un développement suffisant [et permettre] d'installer convenablement les divers services qui en dépendent $\aleph^{59}$. La quiétude des astronomes, la sérénité requise pour leur activité, le nécessaire isolement d'un observatoire thébaïde, restent les arguments mis en avant pour justifier l'enfermement et la claustration. Imposées par Frédéric Petit, ces exigences d'éloignement et de retranchement sont reprises sous la III République par les directeurs de l'établissement savant. Après 1870, la volonté des astronomes de préserver leur espace de recherche s'oppose au développement urbain qui, inexorablement, gagne du terrain. David Aubin a souligné qu'au XIX ${ }^{e}$ siècle, l'Observatoire de Paris « doit réagir et s'adapter au défi posé par l'expansion de la ville » ${ }^{60}$ . L'historien remarque que «toutes les formes de la cité moderne ne conviennent pas à l'observatoire ${ }^{61}$. Toulouse connaît sous la $\mathrm{III}^{\mathrm{e}}$ République une importante expansion urbaine. La ville s'étend de façon anarchique ${ }^{62}$. Le faubourg dans lequel s'insère l'observatoire de la cité garonnaise à la fin du XIXe siècle est donc un territoire morcelé, peu dense, qui s'organise peu à peu le long de voies nouvelles et emprunte au centre-ville les aménagements indispensables à la circulation de la population. Les astronomes doivent composer avec l'irruption des aménagements citadins qui troublent l'isolement souhaité pour la pratique savante et l'examen du ciel. Particulièrement les activités polluantes qui cherchent, elles aussi mais pour d'autres raisons, l'isolement qu'offre la périphérie de Toulouse. Baillaud informe le maire en 1885 qu'un certain Roussille souhaite "établir une fabrique de cuirs vernis (...) à quinze mètres de l'entrée de l'observatoire $»^{63}$. Il remarque que les fabriques de cuirs produisent des émanations gênantes et sont insalubres. Surtout, l'astronome met en avant l'inconcevable cohabitation entre une fabrique polluante et un espace savant: «J'ai le devoir d'attirer votre attention sur l'impossibilité où je me trouverais de protéger les objectifs et les miroirs de nos instruments contre les altérations produites par les émanations d'essences employées à chaud. La perfection de nos instruments dépend d'épaisseurs moindres qu'un dix millième de millimètre, épaisseurs auxquelles rien dans les usages ordinaires n'est comparable ${ }^{64}$. L'observatoire ne peut donc être confondu avec un bâtiment public ordinaire. Les pratiques qui s'y déploient sont étrangères aux autres activités urbaines, la 
finesse des instruments et l'exactitude de leurs mesures défient le sens commun. Dans un environnement jugé hostile, l'astronome fait valoir la spécificité de l'espace qu'il occupe et la singularité de son travail. Il existe une nette « dénivellation des sensibilités » entre l'industriel et l'astronome. La culture de la précision impose une réceptivité exacerbée et une attention sensorielle extrême, adaptées aux instruments scientifiques. Le bruit communément supporté par les habitants du faubourg ne peut convenir aux astronomes qui exigent le silence total (fig. $\mathbf{n}^{\circ} \mathbf{8}$ ).

Figure 8

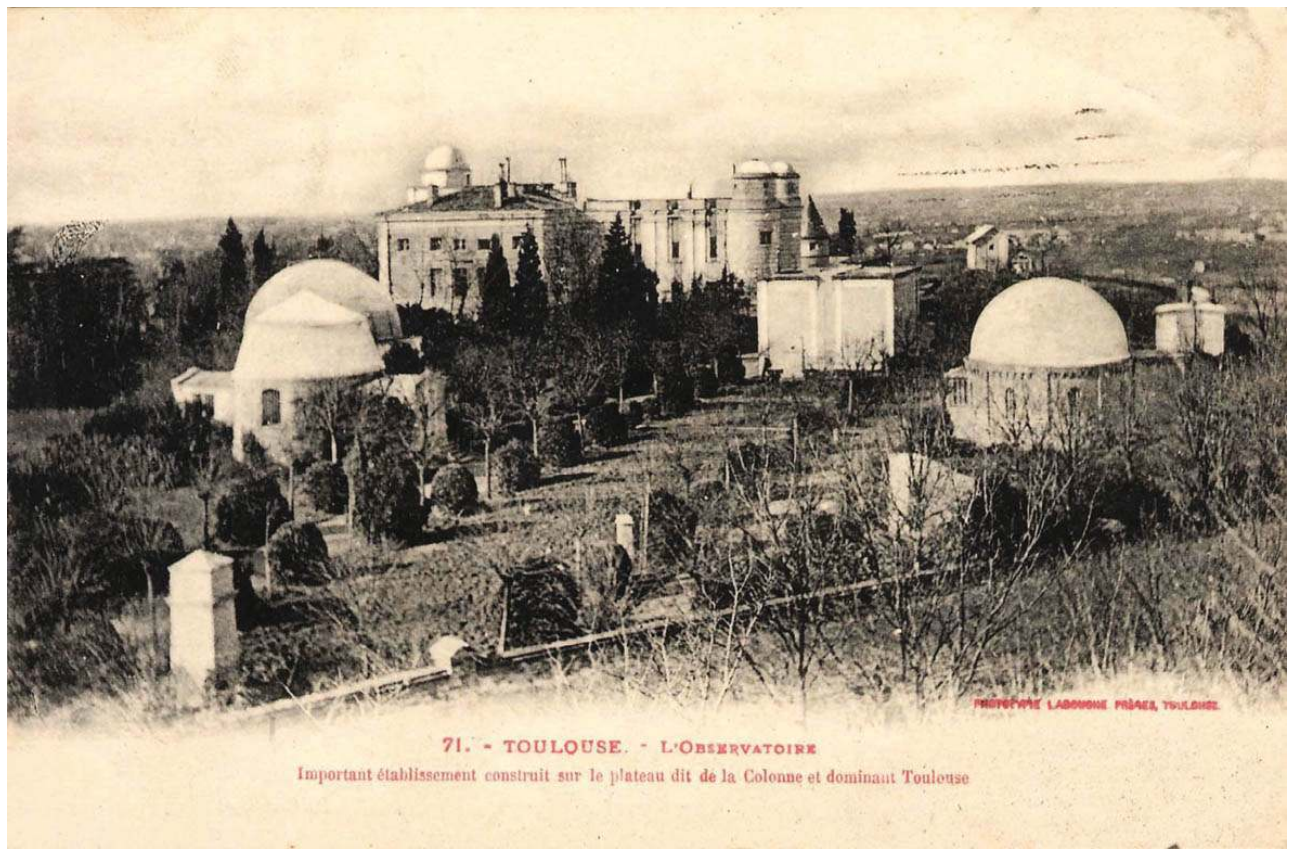

Toulouse. Entrée de l'Observatoire. Carte postale ancienne phototypie ; édition Maison universelle, Toulouse ; imprimerie A. Thiriat, Toulouse. 14 × 9. Archives municipales de Toulouse, cote 9 Fi 863.

On mesure donc combien la structure de l'observatoire, sa topographie et son architecture sont modelées par une pratique savante en mutation à la fin du XIX ${ }^{\mathrm{e}}$ siècle. La dispersion du lieu, son éclatement en coupoles autonomes sont associés à une division stricte des tâches. Dans le même temps, le travail de claustration précédemment entamé se poursuit et s'accentue. L'activité scientifique exige un éloignement des foules, un retrait des activités industrielles et une distance du reste de la ville. La pratique discipline l'espace qui borde l'établissement savant. En somme, l'ordre architectural s'étend au delà du mur de clôture, il déborde l'observatoire pour créer un territoire neutralisé qui protége les astronomes du fracas urbain.

L'histoire de l'observatoire savant, dans sa géographie intime comme dans ses formes monumentales, ne peut être disjointe des évolutions de la science, des changements techniques mais aussi des transformations urbaines. Autour de l'espace savant, de son bâti et de sa topographie, se nouent des enjeux scientifiques, sociaux et politiques qu'une inscription dans un projet patrimonial ne peut ignorer. 


\section{3 - L'observatoire de Toulouse, un espace patrimonial}

l'observatoire de Toulouse. Plusieurs projets muséographiques ont déjà été esquissés ${ }^{65}$; nous limiterons nos propositions au bâti scientifique et à la circulation du public dans l'enceinte du lieu de science. Nous détaillerons donc d'abord les actions patrimoniales en cours sur le site même, dans le quartier de Jolimont, puis nous exposerons quelques pistes pour la mise en valeur de l'architecture de l'observatoire toulousain.

Jusqu'à ce que les astronomes quittent l'établissement savant de Jolimont en 1981, seuls quelques aménagements avaient été ajoutés: un laboratoire d'astrophysique, un radiotélescope et un cercle de pierres ayant servi à préparer la coupole du télescope du pic du Midi ${ }^{66}$. Les scientifiques sont contraints d'abandonner le site historique de l'observatoire, en raison de la pollution lumineuse. L'établissement savant et son personnel s'installent dans de nouveaux locaux sur le campus de l'université à Rangueil. Dès 1980, quelques astronomes avaient entamé une réflexion approfondie sur la sauvegarde de leur patrimoine. Jean-Pierre Brunet et Robert Nadal s'engagent à récupérer et à rassembler l'arsenal instrumental alors en danger. Ils mettent en place une commission "Patrimoine de l'observatoire Midi-Pyrénées » qui gère la protection des fonds anciens d'archives, des clichés de la Carte du ciel et des outils techniques ${ }^{67}$. L'action de cette structure de sauvegarde ne se limite pas aux documents et aux instruments, elle s'étend également à l'ancien observatoire désormais inutilisé. Les objets mobiliers dans les coupoles, immeubles par destination, appartiennent toujours à l'observatoire MidiPyrénées. Il s'agit de la lunette méridienne, de l'astrographe et du télescope de $80 \mathrm{~cm}$ (fig. $\left.\mathrm{n}^{\circ} \mathbf{9}, \mathrm{n}^{\circ} \mathbf{1 0}\right)$. 
Figure 9

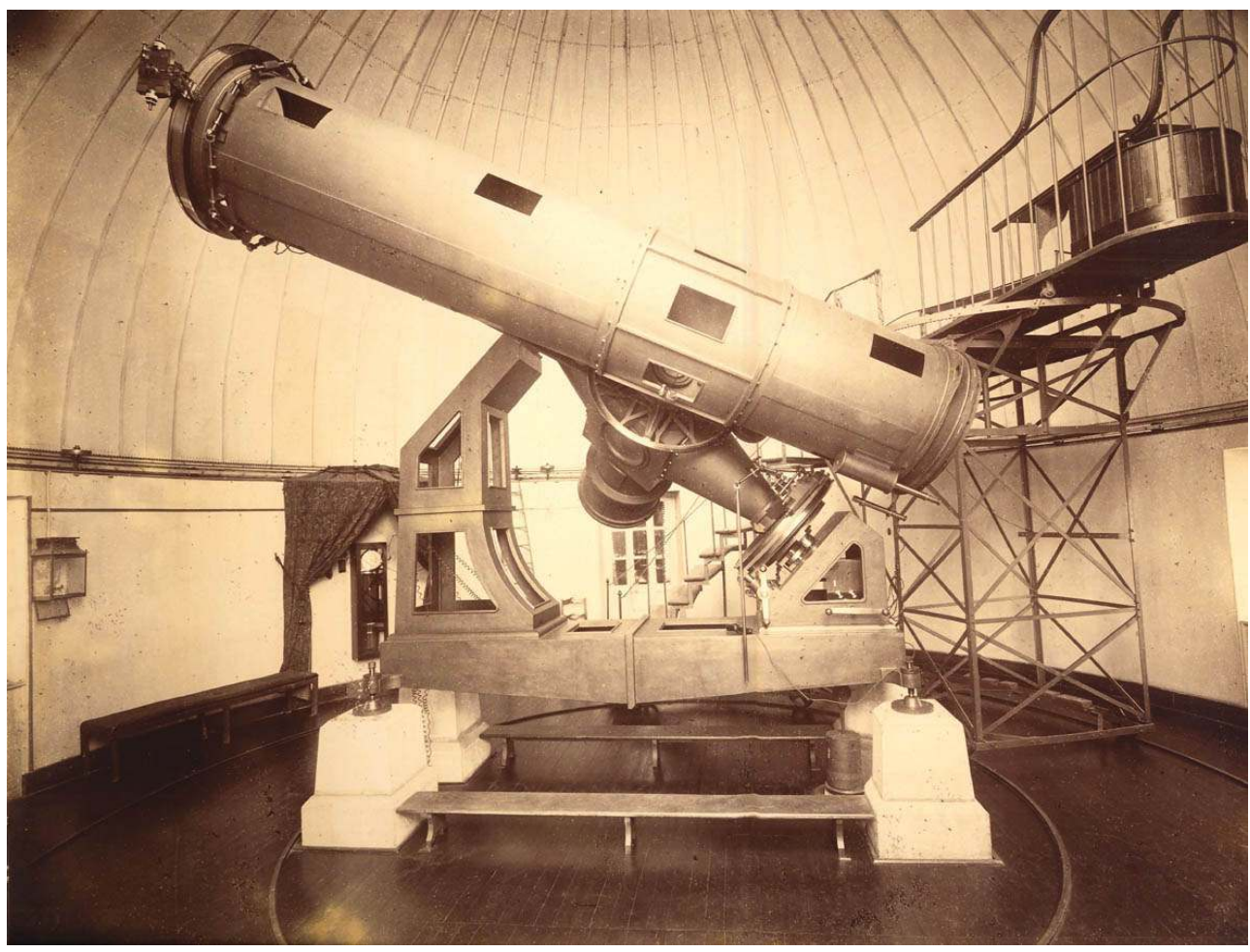

Le grand télescope Gautier. Circa 1900. Photographie 28 × 21, collée sur carton 44 x 34,5. Archives municipales de Toulouse, cote $7 \mathrm{Z}$ (fonds de l'observatoire). 


\section{Figure 10}

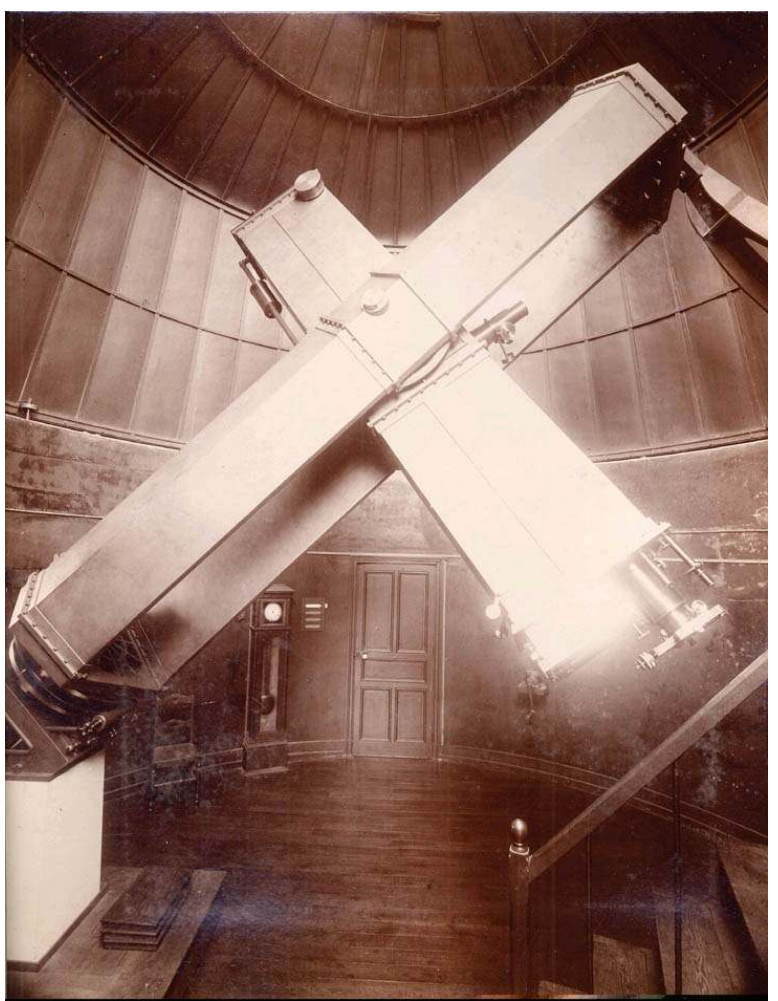

La lunette équatoriale photographique de Gautier dite « Carte du ciel ». Photographie circa 1900, 22,5 $\times 28$, collée sur carton $44 \times 34$. Archives municipales de Toulouse, cote 7 Z (fonds de l'observatoire)

Ils sont classés au titre des Monuments historiques, aucune destruction, modification ou restauration ne doit être réalisée sans l'accord préalable du ministère de la Culture ; les travaux autorisés sont effectués sous la maitrise d'œuvre du conservateur chargé de l'inspection des Monuments historiques. Ces objets sont, de plus, inaliénables (fig. $\mathbf{n}^{\circ} \mathbf{1 1}$ ). 


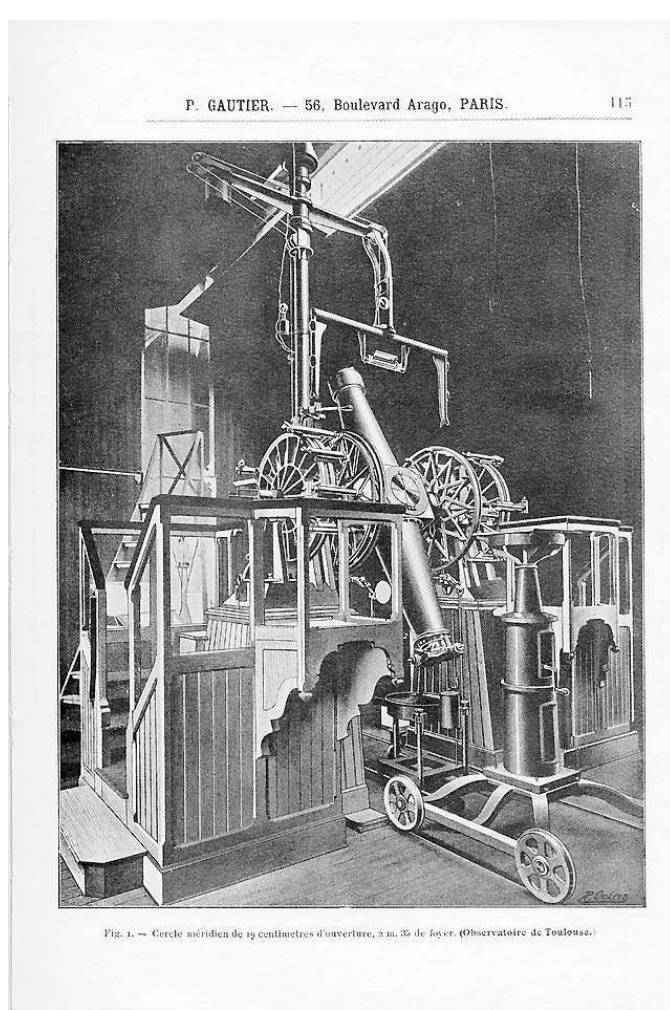

Le cercle méridien de $19 \mathrm{~cm}$ d'ouverture et 2,35 m de distance focale de l'observatoire de Toulouse. Catalogue de la maison Paul Gautier. SYNDICAT DES CONSTRUCTEURS EN INSTRUMENTS D'OPTIQUE \& DE PRÉCISION. L'Industrie française des instruments de précision, 1901-1902. Catalogue. Fac-similé, Paris : Alain Brieux, 1980, p. 115.

En 1986, les membres de la commission régionale du patrimoine historique, archéologique et ethnologique ${ }^{68}$ ont décidé «le classement du matériel et l'inscription à l'inventaire supplémentaire des façades et des toitures du bâtiment principal d'Urbain Vitry et de la porterie, les coupoles de Baillaud et le mur d'enceinte ${ }^{69} »$. Cette protection de l'espace savant et des instruments qu'il contient n'interdit pas un usage actif de ses potentialités scientifiques dans le cadre d'une activité d'amateurs. Depuis les années 1980, plusieurs associations de vulgarisation scientifique se sont installées sur le site de Jolimont $^{70}$, dans les bâtiments existants ou dans des structures préfabriquées. Parmi elles, la Société d'astronomie populaire (SAP) occupe l'ancien laboratoire consacré à l'astrophysique. Elle fait connaître le site par des visites publiques tous les vendredis soirs et par des séances organisées pour les scolaires. Elle utilise encore des instruments appartenant à l'observatoire Midi-Pyrénées (astrographe et télescope de $80 \mathrm{~cm}$ ). Afin d'assurer un usage correct des outils scientifiques mis à la disposition de cette association d'astronomes amateurs, plusieurs conventions ont été signées entre l'observatoire et la SAP. Il y est explicitement stipulé que « la Société d'astronomie populaire de Toulouse est autorisée à utiliser la coupole dite de la «Carte du ciel » de l'ancien observatoire (...) et l'université Paul-Sabatier souhaite continuer à lui apporter son aide scientifique ${ }^{71}$ ».

L'observatoire est aujourd'hui un jardin public de la ville de Toulouse. Il possède donc plusieurs bancs le long d'allées bien dégagées, ainsi qu'un espace de jeux pour les enfants. L'ancien établissement savant est inséré dans le quartier de Jolimont qui est un ensemble urbain très dense, avec de nombreux immeubles autour de l'observatoire. En 2003, la SAP 
et la mairie de Toulouse ont préparé et mis en place un parcours patrimonial dans l'enceinte du monument astronomique. Il a été nécessaire de définir une possible circulation des visiteurs et des promeneurs autour du bâtiment principal et des quatre coupoles. Un premier panneau décrit, à l'entrée, l'ensemble du lieu, donne un aperçu de l'astronomie à Toulouse et propose un circuit de déambulation. Les cinq autres panneaux retracent l'histoire et les mutations du bâtiment principal et des quatre coupoles qui l'entourent. Les informations délivrées sont brèves, pour tenir compte des contraintes du support, sans être allusives, pour ne pas perdre la cohérence historique du lieu.

Cet aperçu des transformations récentes de l'espace savant et des actions patrimoniales engagées nous permet d'avancer quelques propositions de mise en valeur de l'observatoire. Nous esquisserons d'abord un argumentaire théorique sur la lecture historique du monument astronomique ${ }^{72}$.

Michel de Certeau a proposé de distinguer les notions de lieu et d'espace. L'historien expliquait que «l'espace est un lieu pratiqué. Ainsi la rue, géométriquement définie par un urbanisme est transformée en espace par des marcheurs ${ }^{73}$ ». En somme, le lieu n'est qu'une abstraction, une définition désincarnée des surfaces et des formes. L'espace, au contraire, s'emplit des activités quotidiennes et usages courants. Cette distinction nous semble importante car elle permet d'introduire la dimension historique comme outil dialectique permettant de passer du lieu-observatoire (comme l'idée d'un bâtiment savant avec ses fonctionnalités singulières) à l'espace-observatoire (comme matrice des expériences scientifiques et humaines passées). Maurice Halbwachs remarquait qu'un espace reçoit toujours l'empreinte du groupe qui l'occupe. Ainsi, ajoutait le sociologue, «toutes les démarches du groupe peuvent se traduire en termes spatiaux, et le lieu occupé par lui n'est que la réunion de tous les termes. Chaque aspect, chaque détail de ce lieu a lui-même un sens qui n'est intelligible que pour les membres du groupe, parce que toutes les parties de l'espace qu'il a occupées correspondent à autant d'aspects différents de la structure et de la vie de leur société (.... $)^{74}$.

La mise en valeur de l'espace savant s'oppose donc radicalement à une réification patrimoniale. Il s'agit au contraire de restituer la chair historique de l'observatoire, de puiser dans son passé pour retrouver la logique des agencements, des formes et des dispositions. Sophie Forgan explique, dans un récent article, que « les immeubles sont des artefacts en eux-mêmes, créés à grands frais, reflétant le contexte intellectuel et matériel de la société dans laquelle ils ont été édifiés $»^{75}$.

Dans la perspective d'une organisation muséographique incluant l'ensemble des éléments constituant l'observatoire de Toulouse à Jolimont, il est nécessaire de fonder la réflexion patrimoniale sur l'histoire de l'établissement. Il s'agit d'abord du respect des chronologies et des dénominations. Quelques modifications sont donc à envisager. La coupole dite Urbain Vitry, qui contenait l'équatorial, a été érigée par l'entrepreneur Fitte, bien après la mort de Vitry. Cette forme d'hommage est aussi maladroite que fâcheuse. De même, il est faux d'inscrire T 83 sur la plaque fixée sur la coupole du grand télescope. L'instrument a une ouverture utile de $81,2 \mathrm{~cm}^{76}$ (fig. $\mathbf{n}^{\circ} \mathbf{1 2}$ ). 


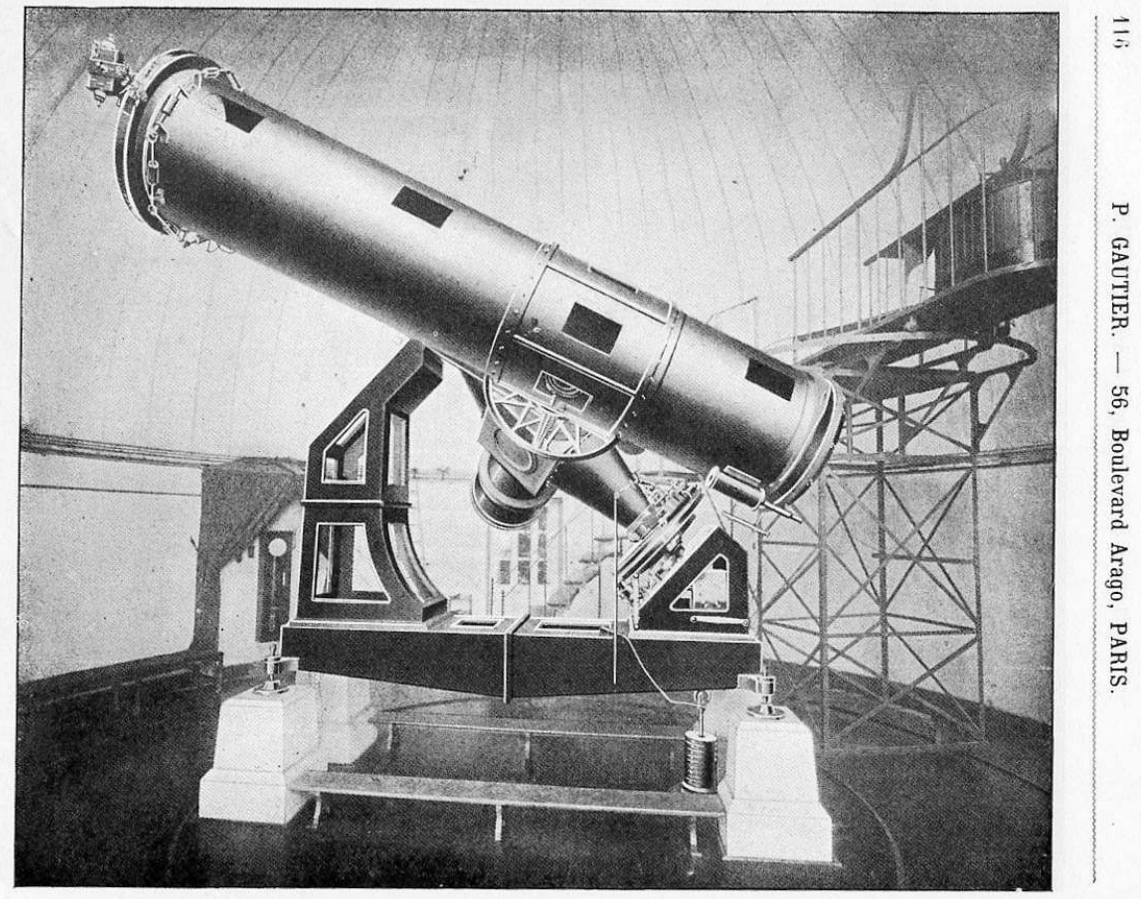

Le télescope de 0,80 m d'ouverture et 4,80 m de distance focale de l'observatoire de Toulouse. Catalogue de la maison Paul Gautier. SYNDICAT DES CONSTRUCTEURS EN INSTRUMENTS D'OPTIQUE \& DE PRÉCISION. L'Industrie française des instruments de précision, 1901-1902. Catalogue. Fac-similé, Paris : Alain Brieux, 1980, p. 116.

Ces erreurs peuvent sembler des détails. Il n'en est rien. Rendre intelligible l'espace savant, c'est restituer à l'architecture et à la topographie du lieu les conditions historiques de leur genèse et de leur transformation. La fragmentation de l'espace, l'éparpillement des coupoles, la disposition particulière de la salle méridienne sont des éléments marquants d'un bâti inhabituel. Les visites ou les présentations muséographiques doivent donc souligner les logiques scientifiques qui, à la fin du XIX ${ }^{e}$ siècle, ont contraint les astronomes à diviser les tâches et à répartir le travail. L'association d'un observateur et d'un instrument renvoie à un principe calqué sur l'organisation industrielle du travail qu'il convient de mettre en avant. De même, le bâtiment d'astrophysique est un bon exemple des mutations des régimes de pratiques qui s'orientent, au milieu du $\mathrm{XX}^{\mathrm{e}}$ siècle, vers une structuration thématique de l'activité scientifique. Il semble également important d'expliquer que l'observatoire était situé à l'écart de la ville, loin du tumulte et de la foule. Les immeubles voisins, l'ouverture du lieu semblent contredire cette intention initiale des astronomes. La mise en valeur du site doit donc rappeler la stricte clôture de l'observatoire jusqu'au départ des observateurs en 1981.

L'histoire de l'observatoire de Toulouse s'inscrit dans l'espace et dans la pierre. Les coupoles et leur agencement répondent à des logiques scientifiques que l'on peut précisément contextualiser. La mise en valeur du site ne doit pas simplement prendre en considération le passé de l'établissement, elle doit se fonder sur la recherche historique. Nous défendons l'idée que la lecture patrimoniale d'un espace savant passe d'abord par le 
dépouillement des archives. L'explication d'une configuration spatiale, la restitution d'une logique architecturale ne sont que les termes d'un travail historique qui peut seul remettre en cause l'évidence d'un lieu dont la pratique a disparu. C'est une entreprise d'archéologie du sens passé que nous proposons comme outil de réflexion patrimoniale.

\section{BIBLIOGRAPHIE}

Aubin, David. The Fading Star of the Paris Observatory in the Nineteenth Century : Astronomers' Urban Culture of Circulation and Observation, OSIRIS, 2003, n 18, p. 79-100.

Baillaud, Benjamin. Notice sur l'état de l'observatoire de Toulouse, Mémoires de l'Académie des Sciences, Inscriptions et Belles-Lettres de Toulouse, 1892, $9^{\text {e }}$ série, T. IV, p. 276-288.

Baillaud, Benjamin. Notices sur le développement successif des études astronomiques à Toulouse, Revue des Pyrénées et de la France méridionale, 1889, n 4, p. 456-474.

Bruand, Yves. Introduction, Urbain Vitry à Toulouse. Catalogue de l'Exposition au Palais des Arts, 20 novembre - 23 décembre 1981. Toulouse : Association des Amis de l'Ecole des BeauxArts de Toulouse, 1981, p. IX-XIII.

Bruand, Yves. L'architecture baroque toulousaine : mouvement original ou maniérisme prolongé, Gazette des Beaux-Arts, 1972, T. LXXX, p. 261-272.

Capella, Marie-Laure. Observatoire de Toulouse. Projet de décoration pour le vestibule et salle de cours, Cinq siècles d'astronomie toulousaine. « Ils observaient les étoiles... ». Toulouse : Archives Municipales de Toulouse, 2002, p. 139.

Capella, Marie-Laure. Urbain Vitry (1802-1863). Un architecte toulousain à l'époque romantique, t. II, Histoire de l'Art. Toulouse : Université de Toulouse II - Le Mirail, 1998, T. I, 397 $f^{\circ}$., T.II, $200 f^{\circ}$., T. III, 189 fo.

Certeau, Michel de. L'invention du quotidien, t. 1, Arts de faire. Paris : Gallimard, 1990, 349 p. Chinnici, Ileana. La Carte du ciel. Correspondance inédite conservée dans les Archives de l'observatoire de Paris. Paris : Observatoire de Paris, 1999, 475 p.

Couder, A. Etude du miroir du télescope de 0m80 de l'observatoire de Toulouse, Annales de l'observatoire astronomique et météorologique de Toulouse, 1935, vol. XI, p. 11-15.

Davoust, Emmanuel. L'observatoire du Pic-du-Midi. Cent ans de vie et de science en haute montagne. Paris : Editions du CNRS, 2000, 552 p.

Eclache, Michèle. L'Observatoire de Toulouse, Ménestral, 1982, n 18, p. 4-9.

Forgan, Sophie. Building the Museum. Knowledge, Conflict, and the Power of Place, Isis, 2005, vol. 96, p. 572-585.

Förster, Robert. Die Sternwarte zu Göttingen im Wandl der Zeiten-Umbauten und Restaurierungen, in Klaus Beuermann (ed.), Grundsätze über die Anlage neuer Sternwarten 
mit Beziehung auf die Sternwarte der Universität Göttingen von Georg Heinrich Borheck. Göttingen : Universitätverlag Göttingen, 2005, p. 46-50.

Freigang, Christian. Architekturhistorische Bemerkungen zur Göttingen Sternwarte, in Klaus Beuermann (ed.), Grundsätze über die Anlage neuer Sternwarten mit Beziehung auf die Sternwarte der Universität Göttingen von Georg Heinrich Borheck. Göttingen :

Universitätverlag Göttingen, 2005, p. 21-25.

Galison, Peter, THOMPSON, Emily (eds.). The Architecture of Science. New York : MIT Press, 1999, 576 p.

Grillot, Solange. Les instruments des observatoires français au XIX ${ }^{\mathrm{e}}$ siècle, L'astronomie, 1986, vol. 100, p. 275-289.

Halbwachs, Maurice. La mémoire collective. Paris : Albin Michel, 1997, 295 p.

Hermann, Dieter B. The history of astronomy from Herschel to Hertzsprung. Cambridge :

Cambridge University Press, 1984, 220 p.

Lalande, Jérôme. Astronomie, tome I. Paris : Imprimerie de P. Didot l'Aîné, 1792, 608 p.

Lamy, Jérôme. Archéologie d'un espace savant. L'observatoire de Toulouse aux XVIII ${ }^{\mathrm{e}}$ et XIX

siècles : lieux, acteurs, pratiques réseaux, Histoire des sciences. Paris : EHESS, T. I : $738 \mathrm{f}^{\circ}, \mathrm{T}$. II : $376 \mathrm{f}^{\circ}$.

Lamy, Jérôme, MOTARD, Béatrice. Inventaire des instruments anciens de l'observatoire MidiPyrénées. Toulouse : Observatoire Midi-Pyrénées, 2002, 303 p.

Lamy, Jérôme, MOTARD, Béatrice. L'inventaire des instruments anciens de l'Observatoire MidiPyrénées : genèse, mise en œuvre et perspectives, La lettre de l'OCIM, 2002, nº 84, p. 26-28.

Lequin, Yves. Les citadins et leur vie quotidienne, in Maurice Agulhon (éd.), Histoire de la France urbaine, T. IV : La ville de l'âge industriel. Le cycle haussmannien. Paris : Le Seuil, 1983. Réédition 1998, p. 285-370.

Paloque, Emile, BOUIGUE, Roger. «L'observatoire de Toulouse. Historique, travaux, outillage. Récents développements ", Annales de l'observatoire astronomique et météorologique de Toulouse, 1948, T. XVIII, p. 5-34.

Petit, Frédéric. Introduction, Annales de l'observatoire de Toulouse, 1862, tome I, p. 1 - 13. Schaffer, Simon. Astronomers Mark Time : Discipline and the Personal Equation, Science in context, 1988, vol. 2, p. 115-145.

Schoebel, Sylvie. Regards sur le patrimoine scientifique et médical. Toulouse : Université Paul Sabatier - Toulouse III, 2001, 38 p.

Van Helden, Albert. Telescope building, 1850-1900, in Owen Gingerich (ed.), The General History of Astronomy, T. IV, Part. A : Astrophysical twentieth-century astronomy. Cambridge : Cambridge University Press, 1984, p. 40-58.

\section{NOTES}

1. GALISON, Peter. Buildings and the subject of science, in Peter Galison, Emily Thompson (eds.), The Architecture of Science. New York : MIT Press, 1999, p. 1.

2. LALANDE, Jérôme. Astronomie, tome I. Paris : Imprimerie de P. Didot l'Aîné, 1792, p. li-lii. 
3. Archives de l'Académie des Sciences, Inscriptions et Belles Lettres de Toulouse (AAST), $80015^{\mathrm{III}}$

${ }^{9}$, Projet pour l'acquisition de la maison de feu M. Garipuy par l'Académie royale des Sciences et Belles Lettres de Toulouse, 20 novembre 1782.

4. Archives Départementales de la Haute-Garonne (ADHG), 20 Toulouse 110, Lettre du ministre des Finances aux administrateurs du département de la Haute-Garonne, 13 thermidor an IV $\left(1^{\mathrm{er}}\right.$ août 1796).

5. Archives Municipales de Toulouse (AMT), AM 6, Lettre d'Alphonse Desplas au Maire de Toulouse, le Comte d'Hargenvilliers, 30 juillet 1825.

6. PETIT, Frédéric. Introduction, Annales de l'observatoire de Toulouse, 1862, tome I, p. 3.

7. AMT, 4D 95, Lettre de Frédéric Petit au maire de Toulouse Armand Perpessac, 13 mai 1839.

8. AMT, 1D 45, Registre des délibérations du conseil municipal de Toulouse (1838-1840), séance du 13 mai 1839, fo 112.

9. AMT, 4D 95, Lettre de François Arago au maire de Toulouse Armand Perpessac, 10 août 1839.

10. AMT, 1D 45, Registre des délibérations du conseil municipal de Toulouse (1838-1839), séance du 9 décembre $1839, f^{\circ} 225 v^{\circ}$.

11. AMT, 4D 95, Lettre de Frédéric Petit au maire de Toulouse Armand Perpessac, 9 décembre 1839.

12. AMT, 4D 95, Lettre de Frédéric Petit au maire de Toulouse Armand Perpessac, 9 décembre 1839.

13. AMT, 4D 95, Lettre de François Arago au maire de Toulouse Armand Perpessac, 10 août 1839.

14. AMT, 4D 95, Lettre de François Arago au maire de Toulouse Armand Perpessac, 10 août 1839.

15. Annuaire administratif, judiciaire et commercial du département de la Haute-Garonne pour l'année 1843. Toulouse : Chez Delsol, 1843, p. 299.

16. Annuaire administratif, judiciaire et commercial du département de la Haute-Garonne pour l'année 1843. Toulouse : Chez Delsol, 1843, p. 299.

17. BRUAND, Yves. Introduction, Urbain Vitry à Toulouse. Catalogue de l'Exposition au Palais des Arts, 20 novembre - 23 décembre 1981. Toulouse: Association des Amis de l'Ecole des Beaux-Arts de Toulouse, 1981, p. XI.

18. AMT, 4D 95, Lettre d'Urbain Vitry au maire de Toulouse Armand Perpessac, 9 novembre 1839.

19. AMT, 4D 95, Lettre de Frédéric Petit au maire de Toulouse Armand Perpessac, 9 décembre 1839.

20. AMT, 4D 95, Lettre de Frédéric Petit au maire de Toulouse Armand Perpessac, 9 décembre 1839.

21. AMT, 4D 95, Lettre de Frédéric Petit au maire de Toulouse Armand Perpessac, 9 décembre 1839.

22. AMT, 4D 95, Lettre de Frédéric Petit au maire de Toulouse Armand Perpessac, 9 décembre 1839.

23. AMT, 4D 95, Lettre de Frédéric Petit au maire de Toulouse Armand Perpessac, 9 décembre 1839.

24. AMT, 4D 95, Lettre de Frédéric Petit au maire de Toulouse Armand Perpessac, 28 juillet 1839. 25. AMT, 4D 95, Achille Leclerc, Rapport fait au conseil des Bâtiments Civils, 8 août 1840.

26. AMT, 4D 95, Rapport de Lambert et Faqué, 26 septembre 1840.

27. AMT, 4D 95, Lettre de Frédéric Petit au maire de Toulouse Pierre Bories, 20 mai 1843.

28. ECLACHE, Michèle. L'observatoire de Toulouse, Ménestral, 1982, nº 18, p. 8.

29. AMT, 4D 95, Requête du Sieur Gillis auprès de l'huissier Pierre Garrigue, huissier au tribunal civil et auditeur de la cour royale de Toulouse, 2 mai 1843.

30. AMT, 4D 95, Etat des dépenses de toute nature faites par la ville pour le nouvel observatoire, 27 novembre 1846.

31. CAPELLA, Marie-Laure. Urbain Vitry (1802-1863). Un architecte toulousain à l'époque romantique, t. II, Histoire de l'Art. Toulouse : Université de Toulouse II - Le Mirail, 1998, p. 40. 
32. CAPELLA, Marie-Laure. Observatoire de Toulouse. Projet de décoration pour le vestibule et salle de cours, Cinq siècles d'astronomie toulousaine. "Ils observaient les étoiles..." ". Toulouse : Archives Municipales de Toulouse, 2002, p. 139.

33. AMT, 4D 95, Lettre de Frédéric Petit au maire Armand Perpessac, 28 juillet 1839.

34. CAPELLA, Marie-Laure. Urbain Vitry (1802-1863). Un architecte toulousain à l'époque romantique, t. I, Histoire de l'Art. Toulouse : Université de Toulouse II - Le Mirail, 1998, p. 190.

35. CAPELLA, Marie-Laure. Observatoire de Toulouse. Projet de décoration pour le vestibule et salle de cours, Cinq siècles d'astronomie toulousaine. "Ils observaient les étoiles..." . Toulouse: Archives Municipales de Toulouse, 2002, p.140. Pour une analyse de ce style architectural, voir : BRUAND, Yves. L'architecture baroque toulousaine : mouvement original ou maniérisme prolongé, Gazette des Beaux-Arts, 1972, T. LXXX, p. 261-272.

36. AMT, 1M 5, Lettre de Frédéric Petit au maire de Toulouse Armand Perpessac, 28 juillet 1839.

37. ADHG, 20 Toulouse 110, Extrait des registres des délibérations du conseil municipal, séance du 14 juillet 1851.

38. AMT, 1M5 Lettre de Frédéric Petit au maire de Toulouse, 3 avril 1846.

39. AMT, 4D 95, Lettre de Frédéric Petit au maire de Toulouse, 24 juin 1846.

40. AMT, 1D 64, Registre des délibérations du conseil municipal (1867-1868), séance du 7 juillet 1868, p. 114.

41. AMT, 4D 95, Ordonnance de Louis-Philippe, 30 mai 1847.

42. Sur ce point, nous nous permettons de renvoyer à notre thèse : LAMY, Jérôme. Archéologie d'un espace savant. L'observatoire de Toulouse aux xviii $^{\mathrm{e}}$ et $\mathrm{xix}^{\mathrm{e}}$ siècles : lieux, acteurs, pratiques réseaux, Histoire des sciences. Paris : EHESS, p. 483-520.

43. HERMANN, Dieter B. The history of astronomy from Herschel to Hertzsprung. Cambridge : Cambridge University Press, 1984, p. 158. "The active industrial production of astronomical instruments grew thoughout the course of the nineteenth century. This was closely connected with the development of measuring instruments, tools, and technical equipment of other sciences ».

44. VAN HELDEN, Albert. Telescope building, 1850-1900, in Owen Gingerich (ed.), The General History of Astronomy, T. IV, Part. A : Astrophysical twentieth-century astronomy. Cambridge : Cambridge University Press, 1984, p. 40. "The period 1850-1900 saw the growth of the refracting telescope to the greatest size it has attained to date, and also the indroduction of the modern reflecting telescope with its metal-coated glass miror $»$.

45. BAILLAUD, Benjamin. Notices sur le développement successif des études astronomiques à Toulouse, Revue des Pyrénées et de la France méridionale, 1889, nº 4, p. 468.

46. AMT, 2R 110, Lettre de Félix Tisserand au ministre de l'Instruction publique, 7 mai 1876.

47. AMT, 2R 110, Lettre de Félix Tisserand au ministre de l'Instruction publique, 7 mai 1876.

48. AMT, 2 R 135, Lettre de Benjamin Baillaud au ministre de l'Instruction publique, 21 mars 1879.

49. PALOQUE, Emile, BOUIGUE, Roger. "L'observatoire de Toulouse. Historique, travaux, outillage. Récents développements", Annales de l'observatoire astronomique et météorologique de Toulouse, 1948, T. XVIII, p. 6-13.

50. BAILLAUD, Benjamin. Notices sur le développement successif des études astronomiques à Toulouse, Revue des Pyrénées et de la France méridionale, 1889, n 4, p. 474.

51. SCHAFFER, Simon. Astronomers Mark Time : Discipline and the Personal Equation, Science in context, 1988, vol. 2, p. 115-145.

52. BAILlAUD, Benjamin. Notice sur l'état de l'observatoire de Toulouse, Mémoires de l'Académie des Sciences, Inscriptions et Belles-Lettres de Toulouse, 1892, $9^{\mathrm{e}}$ série, T. IV, p. 283.

53. AMT, 2R 137, Lettre de Paul Gautier à Benjamin Baillaud, 28 septembre 1885.

54. AMT, 2R 95, Benjamin Baillaud, Rapport sur l'état et le fonctionnement de l'Observatoire de Toulouse en 1890, 14 mars 1891. 
55. AMT, 2R 110, Lettre de Félix Tisserand au ministre de l'Instruction Publique, 7 mai 1876.

56. AMT, 2R 135, Note de Benjamin Baillaud au préfet de la Haute-Garonne, 26 juin 1891.

57. AMT, 1D 114, Registre des délibérations du Conseil municipal (1876), séance du 23 novembre 1876, p. 609.

58. AMT, 1D 114, Registre des délibérations du Conseil municipal (1876), séance du 23 novembre 1876, p. 609.

59. ADHG, 20 Toulouse 110, Arrêté préfectoral du 28 juillet 1893.

60. AUBIN, David. The Fading Star of the Paris Observatory in the Nineteenth Century: Astronomers' Urban Culture of Circulation and Observation, OSIRIS, 2003, $n^{\circ} 18, p .80$. « had to react and adapt directly to the local challenges posed by expanding city ».

61. AUBIN, David. The Fading Star of the Paris Observatory in the Nineteenth Century: Astronomers' Urban Culture of Circulation and Observation, OSIRIS, 2003, n ${ }^{\circ}$ 18, p. 94. " not all forms of the ideals of the modern city suited the observatory ".

62. LEQUIN, Yves. Les citadins et leur vie quotidienne, in Maurice Agulhon (éd.), Histoire de la France urbaine, T. IV : La ville de l'âge industriel. Le cycle haussmannien. Paris : Le Seuil, 1998, p. 304-306.

63. AMT, 2R 100, Lettre de Benjamin Baillaud au maire de Toulouse, 20 avril 1885.

64. AMT, 2R 100, Lettre de Benjamin Baillaud au maire de Toulouse, 20 avril 1885.

65. Voir SCHOEBEL, Sylvie. Regards sur le patrimoine scientifique et médical. Toulouse : Université Paul Sabatier - Toulouse III, 2001, et LAMY, Jérôme, MOTARD, Béatrice. L'inventaire des instruments anciens de l'Observatoire Midi-Pyrénées : genèse, mise en œuvre et perspectives, La lettre de l'OCIM, 2002, n 84, p. 26-28.

66. DAVOUST, Emmanuel. L'observatoire du Pic-du-Midi. Cent ans de vie et de science en haute montagne. Paris : Editions du CNRS, 2000, p. 129-150.

67. LAMY, Jérôme, MOTARD, Béatrice. Inventaire des instruments anciens de l'observatoire Midi-Pyrénées. Toulouse : Observatoire Midi-Pyrénées, 2002, p. 4-32.

68. Cette commission est devenue la commission régionale du patrimoine et des sites (CRPS) en 1999.

69. Extrait du procès-verbal de la COREPHAE, 25 juin 1986.

70. Académie de l'Air et de l'Espace, Sciences et Animation, GAIA, Société d'Astronomie Populaire.

71. Extrait de l'article 5 de la convention relative au transfert de l'observatoire de Toulouse sur le complexe scientifique de Rangueil.

72. Cette problématique a été abordée pour l'observatoire de Göttingen. Voir: FREIGANG, Christian. Architekturhistorische Bemerkungen zur Göttingen Sternwarte, in Klaus Beuermann (ed.), Grundsätze über die Anlage neuer Sternwarten mit Beziehung auf die Sternwarte der Universität Göttingen von Georg Heinrich Borheck. Göttingen : Universitätverlag Göttingen, 2005, p. 21-25, et FÖRSTER, Robert. Die Sternwarte zu Göttingen im Wandl der Zeiten-Umbauten und Restaurierungen, in Klaus Beuermann (ed.), Grundsätze über die Anlage neuer Sternwarten mit Beziehung auf die Sternwarte der Universität Göttingen von Georg Heinrich Borheck. Göttingen : Universitätverlag Göttingen, 2005, p. 46-50.

73. CERTEAU, Michel de. L'invention du quotidien, t. 1, Arts de faire. Paris : Gallimard, 1990, p. 173.

74. HALBWACHS, Maurice. La mémoire collective. Paris : Albin Michel, 1997, p. 196.

75. FORGAN, Sophie. Building the Museum. Knowledge, Conflict, and the Power of Place, Isis, 2005, vol. 96, p. 574. « Buildings are artefacts in themselves, created at considerable expense and reflecting the intellectual and material context of the society in which they were founded ".

76. COUDER, A. Etude du miroir du télescope de $0 \mathrm{~m} 80$ de l'observatoire de Toulouse, Annales de l'observatoire astronomique et météorologique de Toulouse, 1935, vol. XI, p. 11-15. 


\section{RÉSUMÉS}

Cet article explore les aspects historiques et patrimoniaux de l'élaboration et de la mutation de l'observatoire de Toulouse aux XIXe et XXe siècles. De la construction du bâtiment principal à l'éclatement $d u$ lieu en un archipel de coupoles, l'agencement du site révèle les évolutions politiques, scientifiques et techniques qui se lisent dans le bâti. La mise en valeur patrimoniale doit impérativement se fonder sur cette histoire pour permettre une restitution la plus fidèle possible d'un lieu dont l'usage premier nous est étranger. C'est au prix de cette exigence historique que la lecture de l'espace savant dégage le sens passé des configurations et des dispositions spatiales.

\section{INDEX}

Mots-clés : Toulouse, Jolimont, Frédéric Petit, Félix Tisserand, Benjamin Baillaud, François Arago, Paul Gautier, Urbain Vitry, observatoire astronomique, lunette photographique de la carte du ciel, télescope de Foucault

Keywords : Toulouse, Jolimont, Frédéric Petit, Félix Tisserand, Benjamin Baillaud, François Arago, Paul Gautier, Urbain Vitry, sky charts telescope, astronomical observatory, Foucault's Telescope

\section{AUTEURS}

\section{JÉRÔME LAMY}

Post-doctorant CNRS, LISST, UMR 5193, Université Toulouse II. jerome.lamy@laposte.net

\section{BÉATRICE MOTARD}

Professeur des écoles, Niort. beatrice.motard@ac-poitiers.fr 